\title{
Electrostatic double layers as auroral particle accelerators - a problem
}

\author{
D. A. Bryant ${ }^{1, *}$ and G. M. Courtier ${ }^{1, *}$ \\ ${ }^{1}$ formerly at: Rutherford Appleton Laboratory, Chilton, Didcot, Oxfordshire, UK \\ * retired \\ Correspondence to: D. A. Bryant (d.bryant112@btinternet.com)
}

Received: 16 December 2014 - Accepted: 2 April 2015 - Published: 24 April 2015

\begin{abstract}
A search of the Annales Geophysicae database shows that double layers and other quasi-static electric potential structures have been invoked hundreds of times since the year 2000 as being the agents of auroral electron acceleration. This is despite the fact that energy transfer by conservative fields has been known for some 200 years to be impossible. Attention is drawn to a long-standing interpretation of the acceleration process in terms of the dynamic fields of electrostatic waves.
\end{abstract}

Keywords. Space plasma physics (Charged particle motion and acceleration; Electrostatic structures; Wave-particle interactions)

\section{Introduction}

There can be very few instances in the history of science where a notion, so false as to be inconsistent with the very principles upon which the subject is founded, has survived in the literature for more than 60 years. This is, though, exactly what appears to have happened in magnetospheric physics, where potential differences across electrostatic double layers continue to be invoked as the agents of charged particle acceleration. This process is currently referred to 95 times in Annales Geophysicae alone since the year 2000.

\section{Double layers and related potential structures}

As the name implies, an electrostatic double layer is a surface covered with dipoles, each having its axis in the direction of the normal to the surface (Joos, 1951). This configuration produces adjacent potential wells of opposite polarity, giv- ing a relatively strong electric field between the layers and a weaker but more extensive opposing field outside. The potential at any external point is equal to the product of the dipole moment per unit area of the double layer and the solid angle subtended at any point (Hopf, 1948; Joos, 1951). Consequently, the potential difference between any two points at large distances from the double layer tends to zero. The spatial distribution of potential is illustrated in Bryant (1998). The fact that neither a double layer nor any other configuration of charge can result in a net or global change of potential has been known and understood for over 200 years (Poisson, 1811).

The closely related notions of "potential drops" without compensating potential jumps (271 appearances) and of scalar quantities masquerading as vectors, as in "parallel potential" (82), seem similarly to be in breach of the laws of physics. "Field-aligned potentials" (99), "parallel potential drops" (31), as well as depictions of open-ended electrostatic equipotentials, whether "V-shaped" or "U-shaped", are equally enigmatic.

\section{Acceleration by electrostatic waves}

The seeds of a credible theory for the acceleration of auroral electrons were sown when Landau (1946) predicted that electrostatic waves travelling through a plasma would accelerate electrons whose velocities matched those of the waves. The process which quenches the waves and which is often likened to surfing is known as Landau damping. Unfortunately, this process was unknown when the notion of acceleration by potential differences was advanced (Alfvén, 1939, 1940).

The underlying principle is straightforward. Charged particles reflected from a moving potential barrier gain energy 
if they are impelled forward by a faster-moving barrier and lose energy if they run into and are reflected by a more slowly moving barrier. Reflection occurs only if the particles have insufficient energy in the wave frame to negotiate the potential barrier presented by the wave or wave packet. The process is the electrical equivalent of Fermi's mechanism (1949), in which cosmic rays are accelerated or retarded by reflection by the magnetic fields of moving gas clouds. It is also kinematically equivalent to the "sling-shot" effect, whereby spacecraft can gain energy from the gravitational field of a planet in relative motion, to surfing and, more mundanely, to a bat-and-ball collision.

It has long been suggested (Bryant, 1992) that resonant interactions with electrostatic waves could generate the characteristic peak of the auroral electron energy distribution. A numerical stochastic analysis involving energy gains and losses shows that all aspects of auroral electron streams, seen above, below and within the acceleration region could, in principle, be generated by Landau damping of lower hybrid waves (Bryant and Perry, 1995). Lower hybrid waves generated in klystrons and launched from waveguides are employed very successfully in the laboratory to drive currents in tokamaks within the controlled nuclear fusion programme (Fisch, 1987). In the present magnetospheric interpretation, the waves are considered to be continuously generated by positive ions of the plasma sheet through instabilities arising at the boundary with the magnetosphere's tail lobes. A preliminary energy budget suggests that the envisaged process is energetically possible. It is not yet clear, though, whether waves having sufficient amplitude and the necessary phase and group velocities are present in the auroral acceleration region. Much further work is required, both experimental and theoretical, to establish whether this process is indeed responsible.

\section{Conclusions}

It is suggested that, in order to encourage the proper development of the physics of space plasmas, the concept of acceleration by electrostatic potential differences can be safely dismissed. The promising capabilities of the dynamic fields of electrostatic waves warrant further investigation.

Acknowledgements. The authors are grateful to the Referee for suggesting reference to the industrial application of Landau damping.

The topical editor E. Roussos thanks R. A Cairns for help in evaluating this paper.

\section{References}

Alfvén, H.: A theory of magnetic storms and aurorae, Kungl. Sv. Vetenskapsaked. Handl., I, 18, 1939.

Alfvén, H.: A theory of magnetic storms and aurorae, Kungl. Sv. Vetenskapsaked. Handl., II and III, 18, 1940.

Bryant, D. A.: Electron acceleration in space plasmas, Ann. Geophys., 10, 333-343, 1992,

http://www.ann-geophys.net/10/333/1992/.

Bryant, D. A.: Electron acceleration in the aurora and beyond, Institute of Physics Publishing, Bristol and New York, Fig. 1.7, p. 12, 1998.

Bryant, D. A. and Perry, C. H.: Velocity-space distributions of wave accelerated auroral electrons, J. Geophys. Res., 100, 2371123725, 1995.

Fermi, E.: On the origin of cosmic radiation, Phys. Rev. 75, 11691174, 1949.

Fisch, N. J.: Theory of current drive in plasmas, Rev. Mod. Phys. 59, 175-234, 1987.

Hopf, L.: Introduction to the differential equations of physics, Dover Publications, New York, USA, p. 142, 1948.

Joos, G.: Theoretical Physics, Blackie \& Son Ltd, London and Glasgow, p. 271, 1951.

Landau, L. D.: On the vibration of the electronic plasma, J. Phys. (Moscow), 10, 25-34, 1946.

Poisson, S. D.: Traité de mécanique, Courcier, Paris, France, 1811. 